\title{
Fingerprint analysis of Resina Draconis by ultra-performance liquid chromatography
}

\author{
Yudi Xue, Lin Zhu* and Tao Yi ${ }^{*}$
}

\begin{abstract}
Background: Resina Draconis, a bright red resin derived from Dracaena cochinchinensis, is a traditional medicine used in China. To improve its quality control approach, an ultra-performance liquid chromatography (UPLC) fingerprint method was developed for rapidly evaluating the quality of Resina Draconis.

Methods: The precision, repeatability and stability of the proposed UPLC method were validated in the study. Twelve batches of Resina Draconis samples from various sources were analyzed by the present UPLC method. Common peaks in the chromatograms were adopted to calculate their relative retention time and relative peak area. The chromatographic data were processed by Similarity Evaluation System for Chromatographic Fingerprint of Traditional Chinese Medicine software (Version 2004 A) for similarity analysis.

Results: The present UPLC method demonstrated a satisfactory precision, repeatability and stability. The analysis time of the present UPLC method was shortened to $30 \mathrm{~min}$, compared with that of the conventional HPLC method was $50 \mathrm{~min}$. The similarities of the 12 Resina Draconis samples were $0.976,0.993,0.955,0.789,0.989,0.995,0.794,0.994$, $0.847,0.987,0.997,0.986$, respectively, which indicated that the samples were certainly regionally different. The similarities of the 12 samples showed more similar pattern except for samples 4,7 and 9. Such variation in similarity may presumably be attributed to differences in source.

Conclusions: Compared with the conventional HPLC method, the present UPLC method showed several advantages including shorter analysis time, higher resolution and better separation performance. The UPLC fingerprinting established in the present paper provides a valuable reference for the quality control of Resina Draconis.
\end{abstract}

Keywords: Resina Draconis, UPLC, Chromatographic fingerprint, Similarity

\section{Background}

Traditional Chinese medicines (TCMs), which have been used for centuries in China for preventing and treating human diseases, have been gaining more and more global popularity and concern owing to its unique theoretical system and superb efficacy [1]. TCM contains various kinds of herbal medicine and each medicine is composed of complex components which will vary according to many factors including soils, climates, and growth stages [2-4]. Since the therapeutic effects will be influenced by the multiple components of TCM, it is urgent to find a type of quality assessment system to identify species and

*Correspondence: zhulin@hkbu.edu.hk; yitao@hkbu.edu.hk School of Chinese Medicine, Hong Kong Baptist University, Kowloon Tong, Hong Kong Special Administrative Region, People's Republic of China analysis the complex components of TCM. Chromatographic fingerprint, as a main identification method for the comprehensive control of the quality of TCM, becomes the right research objective $[5,6]$. Chinese medicine is multi-component, multi-link, and multi-target and quality control also needs to reflect characteristics of TCM. It's difficult to measure the quality by only a single or a few indexes. TCM fingerprint, based on a systematic research on the chemical composition of TCM, is a kind of comprehensive, quantifiable identification method which is mainly used for the evaluation of the authenticity, superiority and stability of TCM and semi-finished $\mathrm{TCM}$, and conforms to the integrity and fuzziness characteristics of TCM [7].

Recently, chromatographic technologies, such as thinlayer chromatography (TLC), high-performance liquid 
chromatography (HPLC), gas chromatograph (GC) and capillary electrophoresis (CE) have been widely used in TCM fingerprint identification $[5,8,9]$, among which TLC is a traditional method, fast and easy to operate, but with poor resolution. HPLC is the most common fingerprint method with high precision, sensitivity and repeatability. However, HPLC has the disadvantages of long analysis time, low resolution and big solvent consumption. GC is suitable to volatile compounds. CE is often used for the separation and analysis of solubility in water or alcohol soluble ingredient. CE method is well known for its high separation efficiency, fast analysis speed and low cost, however, the retention time is not stable [10, 11]. Therefore, considering the above factors, a method with fast separation and high resolution was expected in the quality control of TCM. Nowadays, UPLC has been gaining popularity in the fast profiling of TCM which is a relatively new technique, and giving new possibilities in liquid chromatography. It managed to save time and solvent consumption [12-16]. As a new type of liquid chromatography, UPLC can significantly improve the degree of separation and detection sensitivity of chromatographic peak, and meanwhile greatly shorten the analysis period, so it is highly suitable for the separation of trace complex mixture and high flux study $[15,16]$. At present, UPLC has been applied in many areas such as metabolomics, food safety, illegal addition of drugs, environmental monitoring, quality control of TCM, etc.

Resina Draconis (also called "dragon's blood"), a bright red resin derived from Dracaena cochinchinensis, is a traditional medicine and regarded as a "panacea of blood activation" in China for long [17-19]. It is clinically used to invigorate blood circulation and applicable in the treatment of many diseases including ischemic heart disease, cerebral arterial thrombosis, blood stasis syndrome and traumatic injuries [20]. Resina Draconis is composed of many constituents, of which flavonoids are the main chemical constituents. Besides, stilbenes, saponins, terpenes, phenols and steroids have also been identified as its constituents [19, 21-23]. In the previous studies, the fingerprint of Resina Draconis has been widely analyzed with chromatographic methods and most of the studies are based on HPLC $[24,25]$. Nevertheless, the methods were quite time-consuming. Recently, a UPLC method was used to evaluation for the quality of Resina Draconis, however, the analysis time of the method was still up to $45 \mathrm{~min}$ [26]. The development of a novel UPLC method remained the primary task for the quality evaluation of Resina Draconis. In this study, a new UPLC method was established for the chromatographic fingerprint validation and quality evaluation of Resina Draconis, aiming to have a better quality control. This experiment investigates the fingerprints of 12 batches of Resina Draconis collected from different regions by UPLC. Meanwhile, the UPLC method is also compared to a HPLC method in order to prove that UPLC method has fast analysis speed, good degree of separation and less required mobile phase, that may provide good reference for the quality control of the dragon's blood.

\section{Experimental}

\section{Materials and reagents}

Twelve batches of Resina Draconis samples were collected from different regions of China for analysis, and the source information was listed in the Additional file 1: Table S1. The authentication of the samples was identified by Dr. YI Tao according to the morphological features, and the voucher specimens were deposited in the School of Chinese Medicine, Hong Kong Baptist University.

Reference compounds of resveratrol, 7,4'-dihydroxyflavone, loureirin A, loureirin B and pterostilbene were provided by the laboratory of quality analysis of TCM, School of Chinese Medicine, Hong Kong Baptist University. The purity of these reference standards was determined to be more than $98 \%$ by normalization of the peak areas detected by using a HPLC-DAD system. Their chemical structures were shown in Fig. 1.

Methanol of analytical grade (Labscan, Bangkok, Thailand) was used for preparation of standards and sample solution. Acetonitrile of chromatographic grade (Labscan, Bangkok, Thailand) and deionized water obtained from a Milli- $Q$ water purification system (Millipore, Bedford, MA, USA) were used for preparation of the mobile phase.

\section{UPLC-PDA instrumentation and conditions}

The UPLC system comprised a $500 \mathrm{~nL}$ flow cell, an auto sampler, and a photodiode array (PDA) detector. The analysis was carried out by an acquity system from waters and an $\operatorname{HSS~C}_{18}$ column $(2.1 \mathrm{~mm} \times 100 \mathrm{~mm}, 1.8 \mu \mathrm{m})$ was used. For UPLC, the mobile phase was a linear gradient consisting of water (A) and acetonitrile (B) in $30 \mathrm{~min}$. The gradient conditions were: $15-20 \%$ (B) at $0-8 \mathrm{~min}$, $20-68 \%$ at $8-30 \mathrm{~min}$. The detection wavelength was set at $280 \mathrm{~nm}$ and the injection volume was $1.0 \mu \mathrm{L}$. The flow rate was $0.3 \mathrm{~mL} / \mathrm{min}$, and the column temperature was maintained at $40{ }^{\circ} \mathrm{C}$ during the separation.

\section{HPLC-DAD instrumentation and conditions}

The HPLC analysis was carried out by an Agilent 1100 series HPLC-diode array detector (DAD) system comprising a vacuum degasser, binary pump, autosampler, thermostated column compartment, and DAD (Agilent, USA), which was used for acquiring chromatograms and ultraviolet (UV) spectra. An Alltima $\mathrm{C}_{18}$ column $(4.6 \mathrm{~mm} \times 250 \mathrm{~mm}, 5 \mu \mathrm{m})$ was used for HPLC analysis. 
<smiles>Oc1ccc(/C=C/c2cc(O)cc(O)c2)cc1</smiles>

Resveratrol<smiles>O=c1cc(-c2ccc(O)cc2)oc2cc(O)ccc12</smiles>

7,4'-Dihydroxyflavone<smiles>COc1ccc(CCC(=O)c2ccc(O)cc2)c(OC)c1</smiles>

Loureirin A<smiles>COc1cc(OC)c(CCC(=O)c2ccc(O)cc2)c(OC)c1</smiles>

Loureirin B<smiles>COc1cc(/C=C/c2ccc(O)cc2)cc(OC)c1</smiles>

Pterostilbene

Fig. 1 Chemical structures of the reference compounds

The mobile phase consisted of water (A) and acetonitrile (B), and the procedure was performed with a gradient program of $23-27 \%(\mathrm{~B})$ at $0-18 \mathrm{~min}, 27-32 \%$ (B) at $18-30 \mathrm{~min}, 32-33 \%$ (B) at $30-35 \mathrm{~min}$ and $33-100 \%$ (B) at $35-50 \mathrm{~min}$. The flow rate was $1 \mathrm{~mL} / \mathrm{min}$. The detection wavelength was set at $280 \mathrm{~nm}$. The column temperature was set at $30{ }^{\circ} \mathrm{C}$. The injection volume of samples and the standard solutions were both $5.0 \mu \mathrm{L}$.

\section{Preparation of the standard solution}

Appropriate amount of resveratrol, 7,4'-dihydroxyflavone, loureirin A, loureirin B and pterostilbene were accurately weighed and dissolved in methanol to obtain the standard solution.

\section{Preparation of the sample solution}

Resina Draconis sample powder (0.1 g) was accurately weighed and put into a $15-\mathrm{mL}$ centrifuge tube. After $10 \mathrm{~mL}$ of methanol was added, the mixture was extracted for $30 \mathrm{~min}$ by ultrasound $(240 \mathrm{~W})$ and centrifuged for $5 \mathrm{~min}$. The operation was repeated once, and the residue was washed with $4 \mathrm{~mL}$ of methanol and then centrifuged for $5 \mathrm{~min}$. The total extracts were combined in a $25-\mathrm{mL}$ volumetric flask, which was then filled up to the calibration mark with methanol. The extracts were then filtered through a microfiltration membrane $(0.20 \mu \mathrm{m})$ to obtain the sample solution.

\section{Validation of the UPLC method}

A Resina Draconis sample (sample 12) was used in the validation test. The precision was determined by injecting the same sample solution for six times in 1 day. The repeatability was determined by analyzing six independently sample solution extracted from Resina Draconis of the same batch. The stability test was evaluated by injecting the same sample solution at $0,2,4,8,12$ and $24 \mathrm{~h}$ after preparation. The 12 batches of Resina Draconis samples from different regions were analyzed, and the chromatograms were recorded.

\section{Data analysis}

The data analysis was processed by the professional software Similarity Evaluation System for Chromatographic Fingerprint of Traditional Chinese Medicine (Version 2004A), which was recommended by the State Food and Drug Administration (SFDA) of China. This software was used to calculate the correlation coefficients of the chromatographic profiles of 12 batches of Resina Draconis samples, and to generate the simulative mean chromatogram (SMC). The similarities of different chromatographic fingerprints were compared with the SMC.

\section{Results and discussion}

Optimization of the preparation methods for the sample solution

This experiment compared the preparation methods of sample solution. By comparing the chromatograms obtained from various extraction solvents, it was found that the chromatographic peak, peak area and base line were relatively steady when methanol was used as extraction solvent. By comparing the ultrasound and reflux extraction, no obvious difference in the efficiency was observed between the two extraction methods, so the ultrasound extraction was adopted. Extraction times and cycles were further optimized, and the results demonstrated that exhausted extraction could be achieved when Resina Draconis sample powder of $0.1 \mathrm{~g}$ was extracted 
with $10 \mathrm{~mL}$ of methanol by means of sonication for $0.5 \mathrm{~h}$, twice.

\section{Optimization of the mobile phase}

Different mobile phase compositions such as methanol-phosphoric acid aqueous solution, acetonitrilephosphoric acid aqueous solution, methanol-water and acetonitrile-water system were compared, and acetonitrile-water system was found to give better separation for the chromatographic peaks at a lower column pressure.

\section{Optimization of the detection wavelength}

Full-wavelength scanning from 190 to $400 \mathrm{~nm}$ was conducted by the PDA detector, and the results showed that the chromatogram at detection wavelength of $280 \mathrm{~nm}$ was abundant in peak information with more obvious characteristics. The five reference components, namely resveratrol, 7,4'-dihydroxyflavone, loureirin $\mathrm{A}$, loureirin $B$ and pterostilbene, were well presented at $280 \mathrm{~nm}$ and the baseline was steady. Thus, the detection wavelength was determined to be $280 \mathrm{~nm}$ eventually.

\section{Optimization of the column temperature}

The effect of the column temperature (25, 30, 40 and $45{ }^{\circ} \mathrm{C}$ ) on the chromatographic peak separation was investigated, and it was found that the resolution of the peaks got better at $40{ }^{\circ} \mathrm{C}$ UPLC, and the best resolution appeared at $30^{\circ} \mathrm{C}$ by HPLC. Thus, 40 and $30^{\circ} \mathrm{C}$ were used by UPLC and HPLC, respectively.

\section{Identification of the common peaks}

The UPLC fingerprints generated by the 12 batches of Resina Draconis samples were analyzed and 10 common peaks were found. Among them, five common peaks were identified by comparing the reference substances, namely resveratrol (peak 1), 7,4'-dihydroxyflavone (peak 2), loureirin A (peak 3), loureirin B (peak 4) and pterostilbene (peak 5).

\section{Comparison of the HPLC and UPLC fingerprints}

The chromatograms of the conventional HPLC and UPLC were compared in Fig. 2. For the conventional HPLC, a complete fingerprint chromatogram of Resina Draconis was obtained in $50 \mathrm{~min}$ at a flow rate of $1.0 \mathrm{~mL} / \mathrm{min}$; but with UPLC, the analysis time was shortened to $30 \mathrm{~min}$ at a flow rate of $0.3 \mathrm{~mL} / \mathrm{min}$. The analysis efficiency of UPLC is higher, which can remarkably shorten the analysis time and reduce the consumption of mobile phase. Compared with HPLC, the elution requirement of UPLC is simpler, the drift
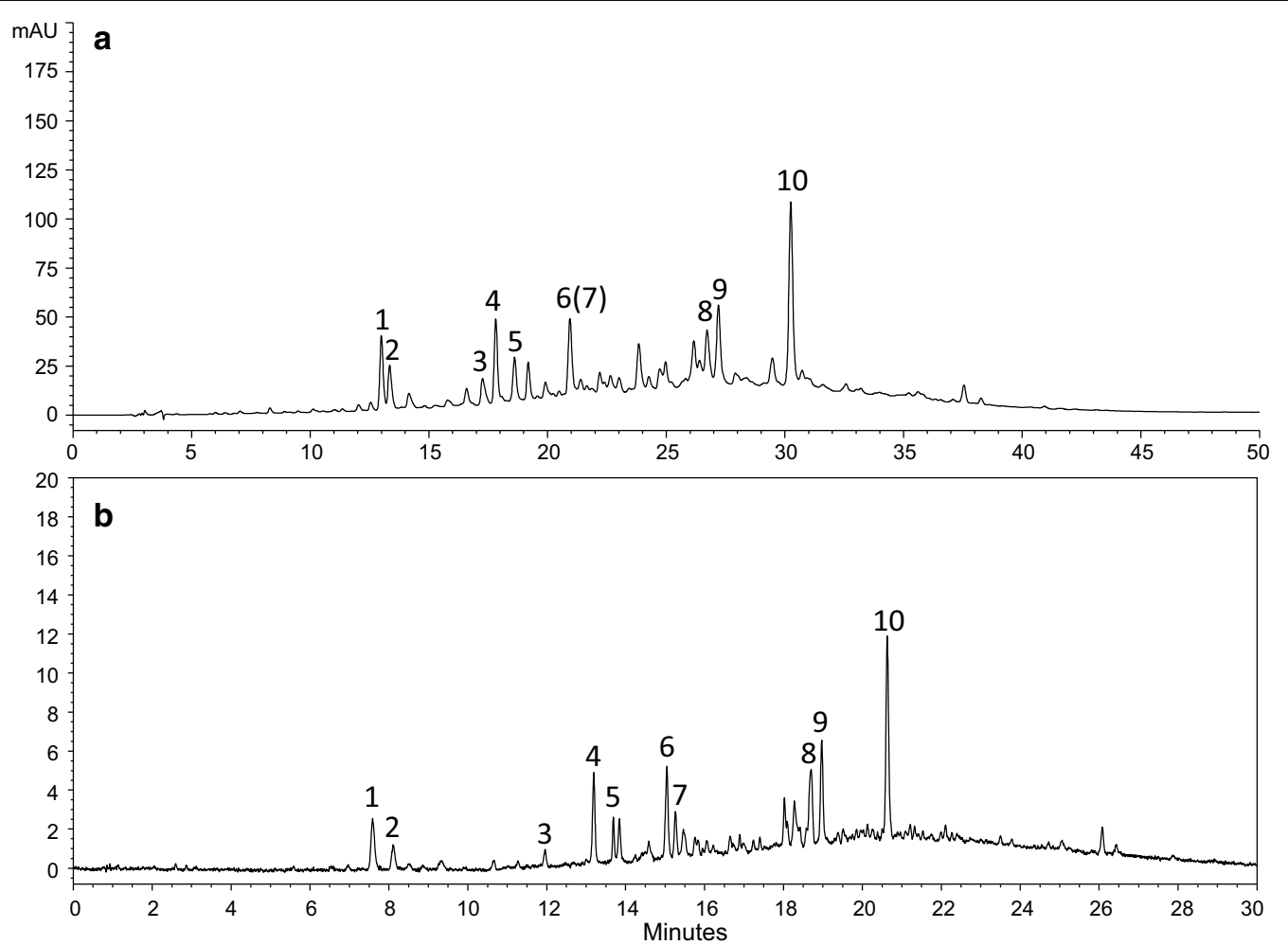

Fig. 2 Resina Draconis (sample 12) on conventional HPLC and UPLC at 280 nm: HPLC (a); UPLC (b). Peak 1 resveratrol; Peak 2 7,4'-dihydroxyflavone; Peak 3 loureirin A; Peak 4 loureirin B; Peak 5 pterostilbene 
time of chromatographic peak is shorter and the peak of the chromatogram is easier to match. UPLC adopts $1.8 \mu \mathrm{m}$ superfine chromatographic column filling while HPLC adopts $5 \mu \mathrm{m}$ chromatographic column filling, so the column efficiency of UPLC is significantly higher than that of HPLC, enabling the separation to be done within $30 \mathrm{~min}$. Compared with the reported UPLC method with separation time of $45 \mathrm{~min}$ in the literature [26], the present UPLC method saved the separation time more than $30 \%$. Owing to the high column efficiency of UPLC, the column length of UPLC is relatively shorter than that of HPLC, which is one reason why UPLC has faster separation speed than HPLC. In addition, although fewer injection volumes were used for UPLC analysis, more and stronger peak signals were obtained. These results indicated that UPLC had superior sensitivity and resolution to the conventional HPLC.

\section{Validation of the UPLC fingerprint method Precision test}

For the precision study, the retention time and peak area of the peak 4 (loureirin B) was chosen as the reference, and the relative retention time (RRT) and relative peak area (RPA) of the ten common peaks of all the samples were calculated. The relative standard deviation (RSD) of the RRT of each common peak was found to be less than $0.05 \%$, and the RSD of the RPA of each common peak was less than $4.68 \%$ (Table 1), which showed the precision of the UPLC fingerprint method was good.

\section{Repeatability test}

The RRT and RPA of the ten common peaks were calculated in the repeatability test. The RSD of the RRT for each peak was less than $0.14 \%$, and the RSD of RPA was less than $4.79 \%$. The two RSD prompted that the repeatability of the UPLC method was satisfied.

\section{Stability test}

For the stability test, the sample solution has been measured at $0,2,4,6,8,12$ and $24 \mathrm{~h}$ after preparation, and then the RRT and RPA were calculated. The RSD of the RRT was found to be less than $0.18 \%$ and the RSD of RPA was less than $4.41 \%$. The results showed that Resina Draconis sample solution was stabile within $24 \mathrm{~h}$.

\section{Similarity analysis}

Using the Similarity Evaluation System for Chromatographic Fingerprint of Traditional Chinese Medicine (Version 2004A), the RRT and RPA of ten common peaks of 12 batches of Resina Draconis samples were calculated, and the results were listed in Table 2, respectively. The RSD of the RRT was found to be less than $0.52 \%$, while the RSD of the RPA were relatively larger. These results indicated that the retention time of the common peaks were consistent among batches, but the contents of the components among batches significantly varied due to the different origin.

The overlapped chromatographic fingerprints from 12 batches of Resina Draconis samples were shown in Fig. 3. The results of the similarity analysis were listed in Table 3. Comparison with the SMC, the similarities of the chromatograms of the 12 samples were $0.976,0.993$, $0.955,0.789,0.989,0.995,0.794,0.994,0.847,0.987$, $0.997,0.986$, respectively, which indicated that Resina Draconis samples from different regions were certainly regionally different, but within a moderate and acceptable range. The similarities of the twelve samples showed more similar pattern except for the samples no. 4, 7 and 9, when The threshold was set to 0.9. This difference in

Table 1 The precision, repeatability and stability of the common peaks in Resina Draconis

\begin{tabular}{|c|c|c|c|c|c|c|}
\hline \multirow[t]{2}{*}{ Peak no. } & \multicolumn{2}{|c|}{ Precision (RSD, \%) } & \multicolumn{2}{|c|}{ Repeatability (RSD, \%) } & \multicolumn{2}{|c|}{ Stability (RSD, \%) } \\
\hline & RRT & RPA & RRT & RPA & RRT & RPA \\
\hline 1 & 0.04 & 4.68 & 0.14 & 3.21 & 0.18 & 0.76 \\
\hline 2 & 0.05 & 0.54 & 0.12 & 3.7 & 0.12 & 2.03 \\
\hline 3 & 0.04 & 0.9 & 0.04 & 1.93 & 0.08 & 0.91 \\
\hline $4(S)$ & - & - & - & - & - & - \\
\hline 5 & 0.02 & 1.46 & 0.02 & 4.5 & 0.01 & 0.63 \\
\hline 6 & 0.01 & 2.2 & 0.03 & 3.21 & 0.02 & 4.41 \\
\hline 7 & 0.03 & 2.89 & 0.01 & 2.78 & 0.01 & 0.52 \\
\hline 8 & 0.02 & 2.9 & 0.04 & 4.79 & 0.04 & 0.5 \\
\hline 9 & 0.02 & 3.95 & 0.04 & 2.63 & 0.04 & 1.62 \\
\hline 10 & 0.02 & 0.53 & 0.04 & 4.33 & 0.04 & 1.01 \\
\hline
\end{tabular}

$R R T$ relative retention time, $R P A$ relative peak area 


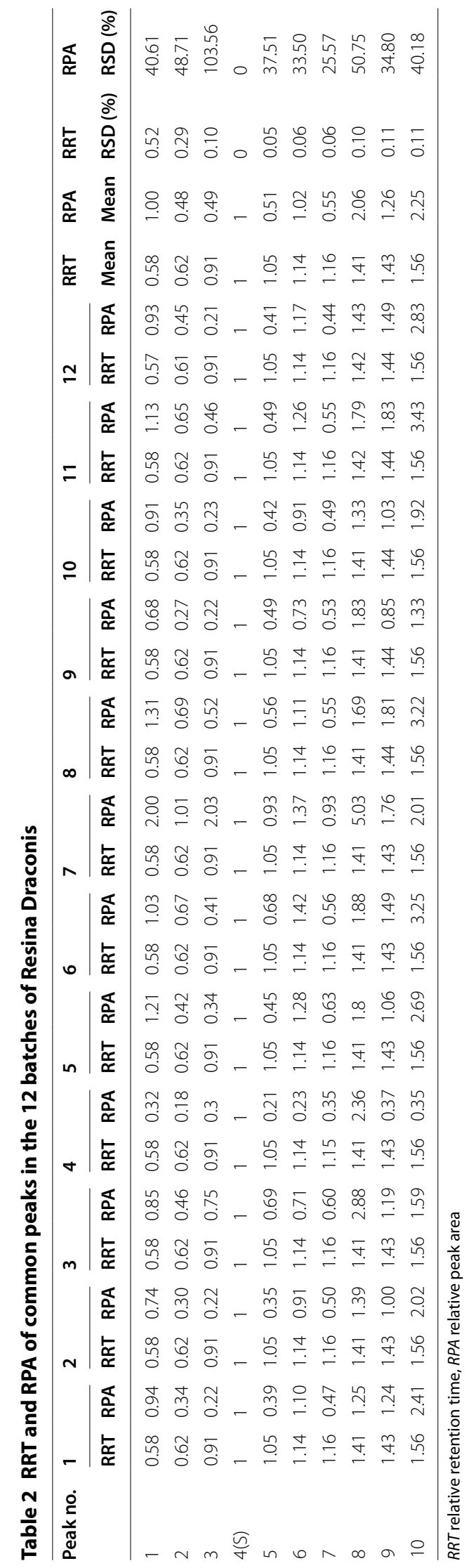




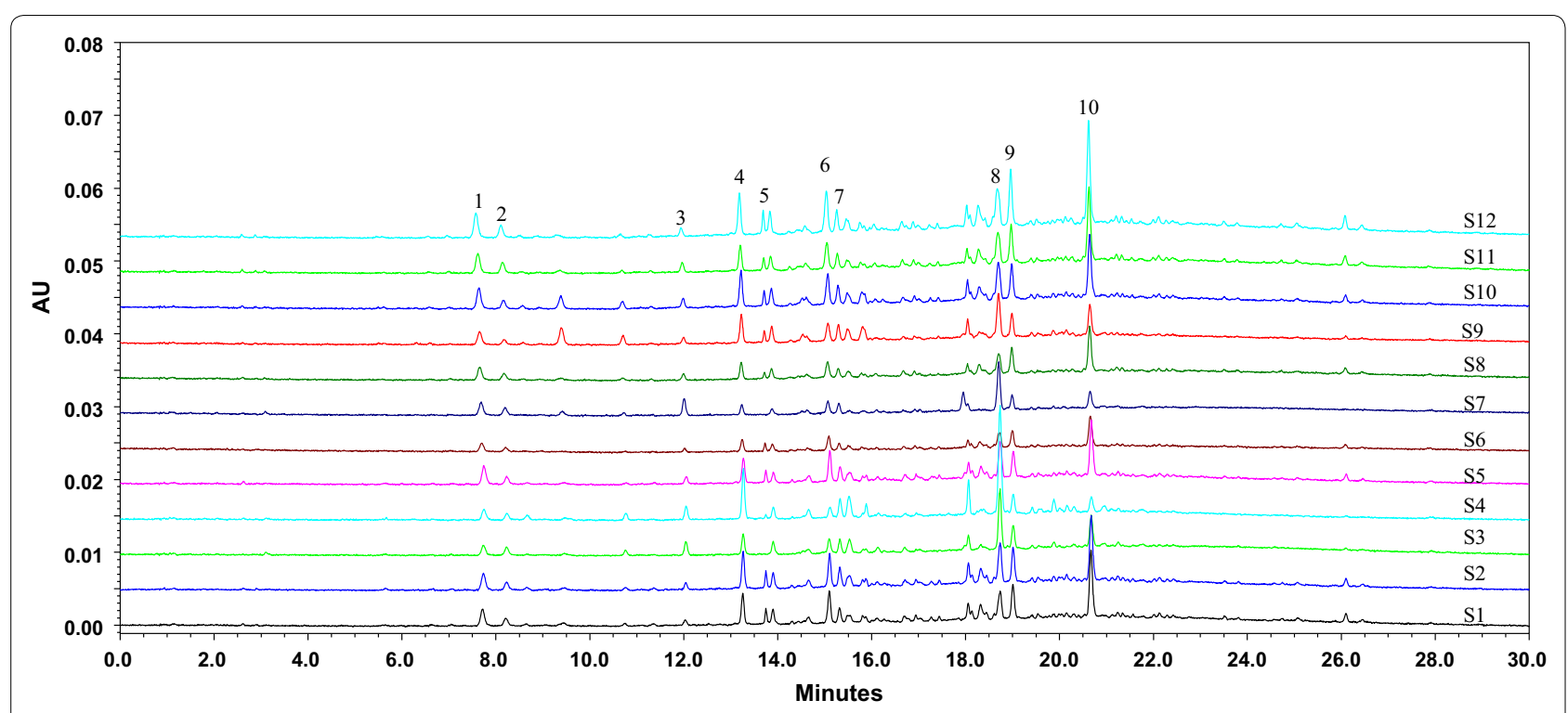

Fig. 3 UPLC fingerprints of 12 batches of Resina Draconis at $280 \mathrm{~nm}$. S1-S12 represents Resina Draconis samples numbered from 1 to 12

Table 3 Similarities of the 12 batches of Resina Draconis

\begin{tabular}{ll}
\hline Sample no. & Similarity \\
\hline 1 & 0.976 \\
2 & 0.993 \\
3 & 0.955 \\
4 & 0.789 \\
5 & 0.989 \\
6 & 0.995 \\
7 & 0.794 \\
8 & 0.994 \\
9 & 0.847 \\
10 & 0.987 \\
11 & 0.997 \\
12 & 0.986 \\
\hline
\end{tabular}

similarity may be due to the difference in the sample origin. The samples 4, 7 and 9 were collected from Guangxi province of China, and the remaining nine batches of samples (the samples 1, 2, 3, 5, 6, 8, 10, 11 and 12) were collected from Yunnan province, China (Additional file 1: Table S1).

The results of similarity analysis showed that the chemical types of Resina Draconis samples from different regions were basically same, however, the relative contents of the each component were various in some of the samples. This finding demonstrated that the present UPLC fingerprint method could not only distinguish the origin, but also evaluate the relative quality of the Resina
Draconis product, which were suitable for the quality control of Resina Draconis.

\section{Conclusion}

A UPLC method for the fingerprinting of Resina Draconis has been established and validated in this study. Compared to the conventional HPLC, the present UPLC method provided a shorter analysis time and higher resolution with good precision, reproducibility and stability. The satisfactory performance of the method was demonstrated through analyzing 12 batches of Resina Draconis samples collected from different regions. To conclude, the UPLC fingerprint method established in the present study was proved to be feasible and reliable, which is extremely helpful in providing a valuable reference for quality control of Resina Draconis and other traditional Chinese medicine.

\section{Additional file}

Additional file 1: Table S1. The source of the tested samples.

\section{Abbreviations}

TCM:Traditional Chinese medicine; UPLC: ultra-performance liquid chromatography; HPLC: high-performance liquid chromatography; RRT: relative retention time; RPA: relative peak area; RSD: relative standard deviation; TLC: thin-layer chromatography; HPLC: high-performance liquid chromatography; GC: gas chromatograph; CE: capillary electrophoresis; PDA: photodiode array; DAD: diode array detector; UV: ultraviolet; SFDA: State Food and Drug Administration; SMC: simulative mean chromatogram.

\section{Authors' contributions}

TY and LZ initiated and designed the study. YDX developed the method and drafted the manuscript. HJ and HLW conducted the sample extraction. All 
authors contributed to data analysis and manuscript finalization. All authors read and approved the final manuscript.

\section{Acknowledgements}

This research was partially supported by the National Natural Science Foundation of China $(81603381,81673691)$ the Guangdong Natural Science Foundation (2014A030313766, 2016A030313008), the Shenzhen Science and Technology Innovation Committee (JCYJ20160518094706544), and the Faculty Research Grant of Hong Kong Baptist University (FRG2/15-16/022).

\section{Competing interests}

The authors declare that they have no competing interests.

\section{Publisher's Note}

Springer Nature remains neutral with regard to jurisdictional claims in published maps and institutional affiliations.

Received: 13 January 2017 Accepted: 14 July 2017

Published online: 24 July 2017

\section{References}

1. Gao HM, Wang ZM, Li YJ, Qian ZZ (2011) Overview of the quality standard research of traditional Chinese medicine. Front Med 5:195-202

2. Yong J, Bruno D, Tu PF, Yves B (2010) Recent analytical approaches in quality control of traditional Chinese medicines - a review. Anal Chim Acta 657:9-18

3. Yi T, Zhu L, Zhu GY, Tang YN, Xu J, Fan JY, Zhao ZZ, Chen HB (2016) HSCCC-based strategy for preparative separation of in vivo metabolites after administration of an herbal medicine: Saussurea laniceps, a case study. Sci Rep 6:33036

4. Yi T, Fang JY, Zhu L, Ji H, Zhang YZ, Zhang XJ, Zhao ZZ, Chen HB (2016) The variation in the major constituents of the dried rhizome of Ligusticum chuanxiong (Chuanxiong) after herbal processing. Chin Med 11:26

5. Christophe T, Bieke D, Yvan VH (2011) Chromatographic separation techniques and data handling methods for herbal fingerprints: a review. Anal Chim Acta 690:148-161

6. Yi T, Chen QL, He XC, So SW, Lo YL, Fan LL, Xu J, Tang YN, Zhang JY, Zhao ZZ (2013) Chen HB (2013) Chemical quantification and antioxidant assay of four active components in Ficus hirta root using UPLC-PAD-MS fingerprinting combined with cluster analysis. Chem Cent J 7:115

7. Li Q, Du SM, Zhang L, Lv CM, Zhou YQ, Zhao Y, Zhang N (2013) Progress in fingerprint technology on Chinese materia medica and prospect of its future development. Chin Tradit Herb Drugs 22:3095-3104

8. Yi T, Tang YN, Zhang JY, Zhao ZZ, Yang ZJ, Chen HB (2012) Characterization and determination of six flavonoids in the ethnomedicine "Dragon's Blood" by UPLC-PAD-MS. Chem Cent J 6:116

9. Custers D, Van Praag N, Courselle P, Apers S, Deconinck E (2017) Chromatographic fingerprinting as a strategy to identify regulated plants in illegal herbal supplements. Talanta 164:490-502

10. Yi T, Zhu L, Peng WL, He XC, Chen HL, Li J, Yu T, Liang ZT, Zhao ZZ, Chen HB (2015) Comparison of ten major constituents in seven types of processed tea using HPLC-DAD-MS followed by principal component and hierarchical cluster analysis. LWT Food Sci Technol 62:194-201

11. Roblova V, Bittova M, Kuban P, Kuban V (2016) Capillary electrophoresis fingerprinting and spectrophotometric determination of antioxidant potential for classification of Mentha products. J Sep Sci 39:2862-2868

12. Jing J, Ren WC, Chen SB, Wei M, Harendra SP (2013) Advances in analytical technologies to evaluate the quality of traditional Chinese medicines. Trac Trends Anal Chem 44:39-45

13. Khan H, Ali J (2015) UHPLC/Q-TOF-MS technique: introduction and applications. Lett Org Chem 12:371-378
14. Chen LN, Song FR, Liu ZQ, Zheng Z, Xing PJ, Liu SY (2012) Multi-residue method for fast determination of pesticide residues in plants used in traditional Chinese medicine by ultra-high-performance liquid chromatography coupled to tandem mass spectrometry. J Chromatogr A 1225:132-140

15. Yi T, Zhu L, Tang YN, Zhang JY, Liang ZT, Xu J, Zhao ZZ, Yu ZL, Bian ZX, Yang ZJ, Chen HB (2014) An integrated strategy based on UPLCDAD-QTOF-MS for metabolism and pharmacokinetic studies of herbal medicines: tibetan "Snow Lotus" herb (Saussurea laniceps), a case study. J Ethnopharmacol 153:701-713

16. Barbero GF, Liazid A, Ferreiro-González M, Palma M, Barroso CG (2016) Fast separation of capsaicinoids from peppers by reversed phase ultraperformance liquid chromatography: comparation with traditional high-performance liquid chromatography methods. Int J Food Prop 19:984-992

17. Shi C, Yu HF, HU WY, Zhang RP (2011) Comparison of the import and Chinese dragon's blood. Prog Mod Biomed 11:4790-4792

18. Yi T, Chen HB, Zhao ZZ, Yu ZL, Jiang ZH (2011) Comparison of the chemical profile and anti-platelet aggregation effects of two "Dragon's Blood"drugs used in traditional Chinese medicine. J Ethnopharmacol 133:796-802

19. Fan JY, Yi T, Sze-To CM, Zhu L, Peng WL, Zhang YZ, Zhao ZZ, Chen HB (2014) A systematic review of the botanical, phytochemical and pharmacological profile of Dracaena cochinchinensis, a plant source of the ethnomedicine "Dragon's Blood". Molecules 19:10650-10669

20. Salam N, Khieu TN, Liu MJ, Vu TT, Chu-Ky S, Quach NT, Phi QT, Narsing Rao MP, Fontana A, Sarter S, Li WJ (2017) Endophytic actinobacteria associated with Dracaena cochinchinensis Lour.: isolation, diversity, and their cytotoxic activities. Biomed Res Int 2017:1308563

21. Pang DR, Su XQ, Zhu ZX, Sun J, Li YT, Song YL, Zhao YF, Tu PF, Zheng J, Li J (2016) Flavonoid dimers from the total phenolic extract of Chinese dragon's blood, the red resin of Dracaena cochinchinensis. Fitoterapia 115:135-141

22. Hao Q, Saito Y, Matsuo Y, Li HZ, Tanaka T (2015) Chalcane-stilbene conjugates and oligomeric flavonoids from Chinese dragon's blood produced from Dracaena cochinchinensis. Phytochemistry 119:76-82

23. Teng Z, Zhang M, Meng S, Dai R, Meng W, Deng Y, Huang L (2015) A comparative study on volatile metabolites profile of Dracaena cochinchinensis (Lour.) S.C. Chen xylem with and without resin using GC-MS. Biomed Chromatogr 29:1744-1749

24. Gao XL, Jiang Q, Wang PJ, Zhang M (2007) RP-HPLC characteristics of dragon's blood. China J Chin Mater Med 32:2025-2027

25. Xu YL, Liang H, Ling Y, Du SW, Wu LH, Gou XJ (2014) Study on HPLC fingerprint of Dracaena cochinchinensis. J Chengdu Univ (Nat Sci) 33:1-3

26. Qin JP, Li JC, Wu JX, Wu SS, Huang WZ, Wang ZZ, Xiao W (2015) Fingerprint analysis of Resina Draconis from different manufactures by UPLC coupled with chemometrics. China J Chin Materia Medica 40:1114-1118

\section{Submit your manuscript to a SpringerOpen ${ }^{\circ}$ journal and benefit from:}

- Convenient online submission

$\checkmark$ Rigorous peer review

- Open access: articles freely available online

- High visibility within the field

- Retaining the copyright to your article

Submit your next manuscript at $\boldsymbol{\nabla}$ springeropen.com 